\title{
Precipitação provável para Barbalha-Ceará, Brasil com uso da distribuição gama
}

\author{
Likely precipitation to Barballha-Ceará, Brazil with use of gama distribution \\ Probable precipitación a Barbalha-Ceará, Brasil utilizando la distribución de gama
}

Recebido: 10/12/2021 | Revisado: 15/12/2021 | Aceito: 21/12/2021 | Publicado: 24/12/2021

\author{
Raimundo Mainar de Medeiros \\ ORCID: https://orcid.org/0000-0001-7361-1281 \\ Universidade Federal Rural de Pernambuco, Brasil \\ E-mail: mainarmedeiros@gmail.com \\ Luciano Marcelo Fallé Saboya \\ ORCID: https://orcid.org/0000-0002-7586-6867 \\ Universidade Federal de Campina Grande, Brasil \\ E-mail: 1saboya @hotmail.com \\ Romildo Morant de Holanda \\ ORCID: https://orcid.org/0000-0001-7945-3616 \\ Universidade Federal Rural de Pernambuco, Brasil \\ E-mail: romildomorant@gmail.com \\ Manoel Viera de França \\ ORCID: https://orcid.org/0000-0003-4973-9327 \\ Universidade Federal Rural de Pernambuco, Brasil \\ E-mail: manoelvieira.ufrpe@gmail.com \\ Moacyr Cunha Filho \\ ORCID: https://orcid.org/0000-0002-3466-8143 \\ Universidade Federal Rural de Pernambuco, Brasil \\ E-mail: Moacyr.cunhafo@ufrpe.br \\ Wagner Rodolfo de Araújo \\ ORCID: https://orcid.org/0000-0001-7203-0338 \\ Universidade Estácio de Sá, Brasil \\ E-mail: wagneraraujops@gmail.com
}

\begin{abstract}
Resumo
Estudos sobre a distribuição pluvial favorecem o planejamento da quantidade d'água necessária para o desenvolvimento das atividades humanas, seja ela pessoal, industrial e agropecuária. Objetiva-se modelar estatisticamente os valores médios mensais de precipitação e estimar as respectivas precipitações em diferentes níveis de probabilidade, através da distribuição gama, para Barbalha - Ceará, no período de 1973 a 2017. O modelo da função probabilística Gama e o teste de aderência dos dados mensais foram avaliados pelo teste de Kolmogorov-Smirnov. As médias fluem entre 3,6 mm (agosto) a 237,4 mm (março). O quadrimestre de maiores índices pluviais registra-se entre janeiro a abril, e o de menores índices ocorrem entre junho a setembro. A oscilação do parâmetro alfa ocorreu de 0,829 (agosto/setembro) a 0,896 (março), o parâmetro beta oscilou entre 0,851(agosto/setembro) a 0,922 (março). Em relação a Probabilidade empírica para ocorrências de totais de chuvas anuais o percentual destaca-se $50 \%$ e $70 \%$ com possibilidades de chuva superior à média histórica.
\end{abstract}

Palavra-chave: Distribuição de probabilidade; Índices pluviais; Anomalias.

\begin{abstract}
Studies on rainfall distribution favor the planning of the amount of water necessary for the development of human activities, whether personal, industrial or agricultural. The objective is to statistically model the monthly mean precipitation values and estimate the respective precipitations at different levels of probability, through the gamma distribution, for Barbalha - Ceará, in the period 1973 to 2017. The Gamma probabilistic function model and the adherence test Monthly data were evaluated by the Kolmogorov-Smirnov test. Means range from $3.6 \mathrm{~mm}$ (August) to $237.4 \mathrm{~mm}$ (March). The four-month period with the highest rainfall is registered between January and April, and the one with the lowest indices occurs between June and September. The oscillation of the alpha parameter occurred from 0.829 (August/September) to 0.896 (March), the beta parameter oscillated between 0.851 (August/September) to 0.922 (March). Regarding the empirical probability for occurrences of total annual rainfall, the percentage stands out $50 \%$ and $70 \%$ with rain possibilities higher than the historical average.
\end{abstract}

Keyword: Probability distribution; Rainfall indexes; Anomalies.

\section{Resumen}

Los estudios sobre la distribución de las lluvias favorecen la planificación de la cantidad de agua necesaria para el desarrollo de las actividades humanas, ya sean personales, industriales o agrícolas. El objetivo es modelar estadísticamente los valores de precipitación media mensual y estimar las respectivas precipitaciones a diferentes 
niveles de probabilidad, a través de la distribución gamma, para Barbalha - Ceará, en el período 1973 a 2017 . El modelo de función probabilística Gamma y la adherencia prueba Los datos mensuales se evaluaron mediante la prueba de Kolmogorov-Smirnov, con medias que oscilan entre 3,6 mm (agosto) y 237,4 mm (marzo). El cuatrimestre con mayor precipitación se registra entre enero y abril, y el de menores índices ocurre entre junio y septiembre. La oscilación del parámetro alfa ocurrió de 0,829 (agosto / septiembre) a 0,896 (marzo), el parámetro beta osciló entre 0,851 (agosto / septiembre) a 0,922 (marzo). En cuanto a la probabilidad empírica de ocurrencia de precipitación total anual, se destaca el porcentaje de $50 \%$ y $70 \%$ con posibilidades de lluvia superiores a la media histórica.

Palabra clave: Distribución de probabilidad; Índices de lluvia; Anomalías.

\section{Introdução}

A região do semiárido brasileiro é considerada a área mais seca do Brasil, predominando índices pluviais anuais com média inferior a $800 \mathrm{~mm}$ ano-1, onde as variabilidades espaciais e interanuais das chuvas são extremamente elevadas (Sá et al., 2010; Silva et al., 2017; Sudene 2021). Além das baixas taxas pluviais, o clima semiárido ainda apresenta altos índices mensais e anuais de insolação e evapotranspiração e forte variabilidade interanual da pluviometria; chuvas concentradas anualmente com estações secas e chuvosas bem definidas; períodos de seca intercalados a períodos chuvosos, com predomínio de anos com chuvas dentro e abaixo da média (Ab Saber 2003; Lucena et al., 2015).

Os estudos das distribuições dos elementos climáticos são necessários para compreender os fenômenos meteorológicos, determinando seus padrões de ocorrência, possibilitando a previsibilidade dos fenômenos climáticos de uma área, sendo uma ferramenta de grande importância para o planejamento e gestão de inúmeras atividades humanas que envolvam recursos hídricos (Dourado Neto et al., 2005).

A quantidade de informações e/ou de dados disponíveis para análise aumenta rapidamente, disponibilizando aplicações de novos modelos de distribuições de probabilidade para melhor descrever as especificidades de cada fenômeno/experimento estudado, principalmente para o elemento precipitação.

Estudiosos de diversas áreas cientificas vêm utilizando-se da ferramenta estatística, para caracterização de determinados fenômenos, no caso dos índices pluviais de uma região, busca-se modelos de distribuição probabilísticas que melhor se adaptam aos conjuntos de dados analisados (Assis et al., 1996, Moura et al., 2001, Vieira et al., 2010, Dourado Neto et al., 2014, Mazucheli et al., 2019). Entre os diferentes modelos de distribuições probabilísticas frequentemente aplicados a modelagem de dados pluviais temos a distribuição Binomial; a distribuição Weibull, a distribuição de Gauss e a distribuição Gama.

Alterações nos índices pluviais vêm ocasionando eventos extremos como inundações, alagamentos, enchentes e secas, uma vez que as variáveis climáticas flutuam (Silva 2013), o obrigando-nos a buscar modelos probabilístico de previsibilidade do regime pluviométrico, auxiliando ações de planejamento de atividades agrícolas, hidrológicas, recursos hídricos, armazenamento e represamento d'água para abastecimento humano e animal e geração de energia, em razão dos grandes impactos socioeconômicos e ambientais no mundo (Ávila et al., 2009; Silva et al., 2013; Uliana et al., 2013; Moura et al., 2015).

A caracterização das variabilidades pluviais nas mudanças climáticas tem sido preocupação de toda a comunidade cientifica e técnica, que busca desenvolver ferramentas capazes de subsidiar ações de planejamento para atividades agropecuárias, abastecimento humano e animal e geração de energia e lazer.

Objetiva-se modelar estatisticamente os valores médios mensais de precipitação e estimar as respectivas precipitações prováveis em diferentes níveis de probabilidade utilizando-se da distribuição gama para Barbalha - Ceará, entre 1973 e 2017.

\section{Material e Métodos}

Barbalha se posiciona na região sul do estado do Ceará, estando inserida na região semiárida brasileira e localiza-se na latitude de $7^{\circ} 18^{\prime}$ Sul, longitude de $39^{\circ} 18^{\prime}$ Oeste, a $409 \mathrm{~m}$ de altitude. (Figura 1). 
Figura 1. Posicionamento de Barbalha na região sul do Ceará.

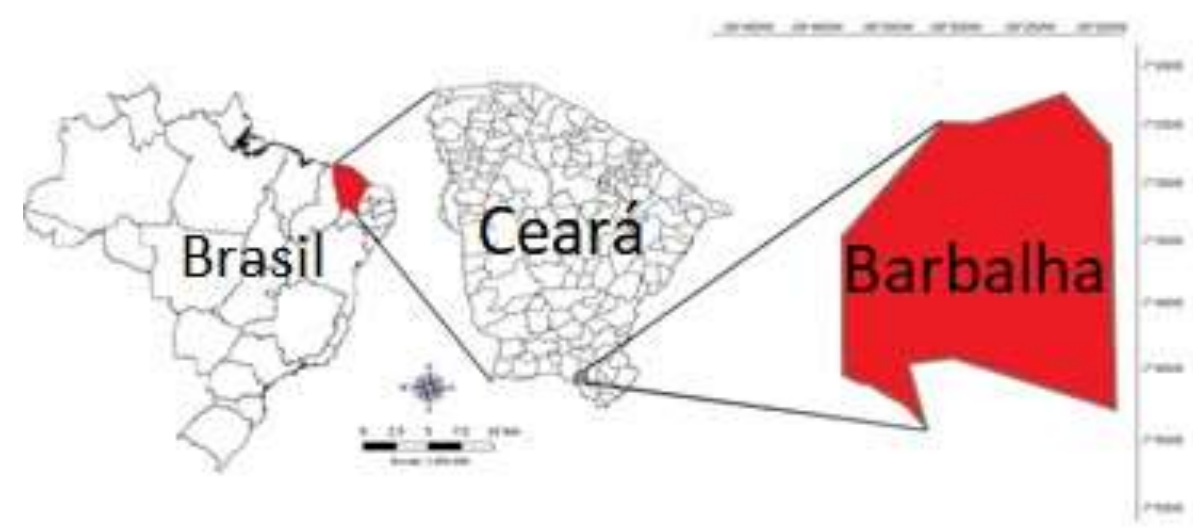

Fonte: Saboya (2021).

O regime pluviométrico possui distribuição irregular, característico do Nordeste brasileiro (NEB), concentra quase todo seu índice pluvial em cinco meses no período chuvoso, de dezembro a abril. Os fatores provocadores de chuva são formações de linhas de instabilidade, intensificação dos ventos alísios de sudeste/nordeste, aglomerados convectivos, troca de calor sensível por latente e vice-versa, orografia, contribuições de formação de vórtices ciclônicos de ar superior e tendo como principal sistema o posicionamento da Zona de Convergência Intertropical (ZCIT) (Medeiros, 2016).

Segundo Thornthwaite (1948) Barbalha - CE tem o clima B $2{ }_{2} A^{\prime} a '$ ', megatérmico com nenhuma deficiência hídrica. Na classificação de Köppen o clima é do tipo “As”, tropical com estação seca de verão. (Alvares et al., 2014).

Os parâmetros Alfa $(\alpha)$ e Beta $(\beta)$ da distribuição Gama foram estimados com o uso de planilha eletrônica e aferidos pelo teste Kolmogorov-Smirnov, ao nível de 5\% significância (Wilks 2006).

A estimativa dos índices pluviais mensais prováveis foi determinada para os níveis de 10, 20, 30, 40, 50, 60, 70, 75, 80, 90 e 95\% de probabilidade utilizando o modelo Gama incompleta (Thom, 1958), em que a função $\mathrm{F}(\mathrm{x})$ é dada pela equação 1.

$f(x)=\frac{1}{\beta^{\alpha} \tau(\alpha)} X^{\alpha-1} e^{\frac{X}{\beta}}$

Onde:

$\beta$ - parâmetro de escala (mm);

$\alpha$ - parâmetro de forma (adimensional);

e - base do logaritmo neperiano;

$\mathrm{X}$ - Precipitação (mm);

$\Gamma(\alpha)$ - função Gama.

A distribuição Gama $\Gamma(\alpha)$ foi calculada utilizando-se a equação 2.

$\tau(\alpha)=\int_{0}^{\alpha} X^{\alpha-1} e^{-X} d(X)$

A distribuição acumulada Gama $F(x)$ foi determinada pela equação (3).

$f(x)=\frac{1}{\beta^{\alpha} \tau(\alpha)} \int_{o}^{X} X^{\alpha-1} e^{\frac{-X}{\beta}} d(X)$

Os elementos de ajuste da função de distribuição Gama, alfa $(\alpha)$ e beta $(\beta)$, foram estimados pelo método da máxima verossimilhança, com a aproximação polinomial para o parâmetro ô proposto por Greenwood et al., (1960) apud Wilks (2006), através das equações 4 e/ou 5. 
$\hat{\alpha}=\frac{0,5000876+0,1648852 A-0,0544274 A^{2}}{A}, 0 \leq \mathrm{A} \leq 0,5772 ;$ ou

$\hat{\alpha}=\frac{8,898919+9,059950 A+0,9775373 A^{2}}{17,79728 A+11,968477 A^{2}+A^{3}}, 0,5772 \leq \mathrm{A} \leq 17,0$.

Calculando-se A pela equação 6.

$A=\ln \bar{X}-\frac{1}{n} \sum_{j=1}^{n} \ln X j$

$X=$ Valores observados

$\bar{X}=$ Média dos valores observados, e

$\hat{\beta}$ Dada pela equação 7 .

$\hat{\beta}=\frac{\bar{X}}{\alpha}$

Em seguida verificou-se através do teste Kolmogorov-Smirnov (KS) ao nível de significância de 5\% a adequabilidade do ajuste adotado em cada mês (WARRICK et al., 1980).

\section{Resultados e Discussões}

No Quadro 1 observa-se os coeficientes de ajuste alfa $(\alpha)$ e beta $(\beta)$ para o modelo de distribuição Gama em BarbalhaCE, entre 1973 e 2017.

A oscilação do parâmetro alfa ocorreu de 0,829 (agosto/setembro) a 0,896 (março), segundo Botelho et al, (1999) a variação de alfa $(\alpha)$ relaciona-se com a assimetria mensal, significando que a assimetria é inversamente proporcional a $\alpha$. $O$ parâmetro beta $(\beta)$ oscilou entre 0,851 (agosto/setembro) a 0,922 (março). O maior índice mensal $\beta$ registrou-se em março, mês que registrou maior índice pluvial, com 237,4 mm e desvio padrão de 115,4 mm.

Quadro 1. Coeficientes de ajuste da função de distribuição Gama para Barbalha-CE entre 1973 e 2017.

\begin{tabular}{|c|c|c|c|c|}
\hline Parâmetros/meses & Alfa & Beta & $\begin{array}{c}\text { Precipitação média } \\
\text { mensal }(\mathrm{mm})\end{array}$ & Desvio padrão (mm) \\
\hline Janeiro & 0,877 & 0,902 & 179,3 & 101,3 \\
\hline Fevereiro & 0,881 & 0,906 & 200,2 & 109,3 \\
\hline Março & 0,896 & 0,922 & 237,4 & 115,4 \\
\hline Abril & 0,866 & 0,891 & 181,3 & 114,5 \\
\hline Maio & 0,837 & 0,86 & 59,4 & 31,3 \\
\hline Junho & 0,834 & 0,856 & 19,2 & 37,6 \\
\hline Julho & 0,83 & 0,852 & 17,9 & 7,7 \\
\hline Agosto & 0,829 & 0,851 & 3,6 & 11,6 \\
\hline Setembro & 0,829 & 0,851 & 6,7 & 34,2 \\
\hline Outubro & 0,83 & 0,853 & 22,6 & 39,4 \\
\hline Novembro & 0,832 & 0,855 & 41,1 & 70,4 \\
\hline Dezembro & 0,839 & 0,862 & 81,6 & \\
\hline
\end{tabular}

Fonte: Saboya (2021).

Na Tabela 1 observa-se os valores do teste de Kolmogorov-Smirnov (Ks) a 5\% de probabilidade para Barbalha-CE, constatou-se que a distribuição de Gama, com os respectivos valores de alfa e beta, apresentados na Tabela1, possibilitou ajustes satisfatório para a estimativa dos índices pluviais mensais. Todos os valores calculados, referentes a cada mês, foi inferior ao valor crítico, significando que ajuste obtido está adequado. 
Tabela 1. Valores do teste de Kolmogorov-Smirnov (Ks) a 5\% de probabilidade para Barbalha-CE, entre 1973 e 2017.

\begin{tabular}{|c|c|c|c|c|c|c|c|c|c|c|c|c|}
\hline \multirow[b]{2}{*}{ D crítico } & \multicolumn{12}{|c|}{ Valores de D observados } \\
\hline & Jan & Fev & Mar & Abr & Mai & Jun & Jul & Ago & Set & Out & Nov & Dez \\
\hline 0,203 & 0,040 & 0,055 & 0,050 & 0,043 & 0,051 & 0,130 & 0,040 & 0,130 & 0,130 & 0,130 & 0,130 & 0,130 \\
\hline
\end{tabular}

Fonte: Saboya (2021).

Na Tabela 2 têm-se os totais pluviométricos mensais $(\mathrm{mm})$ relativos aos níveis de probabilidade de 10, 20, 30, 40, 50, 60, 70, 75, 80, 90 e 95\% para Barbalha-CE, ajustados com a distribuição Gama, referentes ao período de 1973 a 2017.

Tabela 2. Precipitação provável (mm) em diferentes níveis de probabilidade (\%), com uso da distribuição probabilidade Gama, para Barbalha-CE, para o período entre 1973 e 2017.

\begin{tabular}{|c|c|c|c|c|c|c|c|c|c|c|c|c|}
\hline \multirow[b]{2}{*}{ Nível (\%) } & \multicolumn{12}{|c|}{ Precipitação provável (mm) } \\
\hline & Jan & Fev & Mar & Abr & Mai & Jun & Jul & Ago & Set & Out & Nov & Dez \\
\hline 10 & 67,5 & 78,4 & 104,1 & 58,7 & 3,4 & 0,4 & 0,0 & 0,0 & 0,0 & 0,0 & 0,4 & 7,0 \\
\hline 20 & 93,2 & 106,9 & 136,8 & 85,3 & 8,9 & 1,6 & 0,1 & 0,0 & 0,0 & 0,3 & 1,8 & 16,0 \\
\hline 30 & 115,7 & 131,5 & 164,4 & 109,1 & 16,1 & 3,4 & 0,4 & 0,0 & 0,0 & 1,0 & 4,7 & 26,5 \\
\hline 40 & 137,6 & 155,5 & 190,9 & 132,8 & 25,0 & 5,9 & 1,4 & 0,1 & 0,1 & 2,6 & 9,3 & 39,1 \\
\hline 50 & 160,5 & 155,5 & 190,9 & 132,8 & 25,0 & 5,9 & 1,4 & 0,1 & 0,1 & 2,6 & 9,3 & 39,1 \\
\hline 60 & 185,8 & 207,9 & 247,8 & 186,2 & 50,4 & 14,3 & 7,0 & 0,8 & 0,8 & 10,8 & 26,7 & 73,0 \\
\hline 70 & 215,8 & 240,3 & 282,5 & 219,8 & 69 & 21,2 & 13,7 & 1,9 & 1,9 & 19,5 & 42,2 & 97,5 \\
\hline 75 & 233,6 & 259,6 & 303,0 & 240,1 & 81,9 & 25,7 & 19,8 & 3,0 & 3,0 & 26,0 & 52,9 & 113,1 \\
\hline 80 & 254,6 & 282,2 & 326,9 & 264,1 & 97,3 & 31,6 & 26,3 & 4,5 & 4,5 & 34,8 & 66,9 & 132,4 \\
\hline 90 & 315,6 & 347,7 & 395,7 & 334,3 & 146,3 & 50,8 & 53,7 & 10,9 & 10,9 & 66,5 & 114,3 & 192,7 \\
\hline 95 & 372,4 & 408,5 & 459,9 & 400,5 & 196,4 & 71,1 & 53,7 & 19,1 & 19,1 & 102,8 & 165,8 & 253,6 \\
\hline
\end{tabular}

Fonte: Saboya (2021).

Os níveis de probabilidade se referem à probabilidade de ocorrência de uma lâmina de excedente, sendo assim, o aumento na confiabilidade da estimativa resulta no decréscimo da lâmina mínima a precipitar.

Observa-se que, para os níveis de probabilidade de 75\% e 95\%, detectaram-se os maiores valores pluviais efetivos, visto que estes valores estão diretamente relacionados com a precipitação provável. No nível de 75\% os índices pluviais fluem entre 2,95 mm (agosto/setembro) a $303 \mathrm{~mm}$ em março. Janeiro, fevereiro e abril com índices maiores que $200 \mathrm{~mm}$, dezembro registrou precipitação provável de 113,1 mm. Entre maio, junho, julho, outubro e novembro os índices pluviais oscilaram entre 19,8 mm a $81,9 \mathrm{~mm}$.

Nos níveis de $90 \%$ e $95 \%$ registram-se precipitações prováveis inferiores a $54 \mathrm{~mm}$ e $72 \mathrm{~mm}$ respectivamente. Destacamse janeiro, fevereiro e abril com chuvas prováveis menores de $100 \mathrm{~mm}$ para os níveis de $10 \%$ e 20\%. Para os níveis de 10 , 20, 30, 40, 50, 60, 70, 75, 80, 90 registram índices pluviais irregulares inferiores a $100 \mathrm{~mm}$. Os estudos de (Silva et al., 1988; Bernardo 1989) vem a corroboram com os resultados aqui discutidos.

Alterações nas cotas pluviais, enchentes, alagamentos, inundações, secas e a crescente necessidade do conhecimento do regime pluviométrico para a realização de planejamento e desenvolvimento de atividades agropecuárias, vêm causando impactos sócios econômicos e ambientais no Globo. Essas variações climáticas oscilaram significativamente com o tempo, com anos mais secos e alternados por períodos de chuvas intensas, similarmente observado com nossos dados. O problema é recursivo, uma vez que a pluviometria não é pontual (Silva 2013).

Na Figura 2 observa-se a distribuição pluvial anual, média climatológica e sua anomalia para Barbalha Ceará entre 1973-2017. Com precipitação histórica em torno de 1125 mm, observam-se as flutuações anuais para os maiores valores pluviais 
com $2000 \mathrm{~mm}$ (1985), $19891520 \mathrm{~mm}, 1966$ (420 mm), 2004 (550 mm) e os anos de 2008 e 1011 com $560 \mathrm{~mm}$ e $510 \mathrm{~mm}$ respectivamente, tais variabilidades pluviais estão em corroboração com os estudos de (Marengo et al. 2015; Marengo et al. 2008; IPCC 2014). Com um pico de mínimo valor pluvial registrado no ano de 1981; 1982 e 2013 com 578,2 mm; $272 \mathrm{~mm}$ e $595 \mathrm{~mm}$ respectivamente estas oscilações tem influencias dos sistemas pluviais atuante na atmosfera local e regional. (Medeiros et al., 2020).

Figura 2. Distribuição da precipitação anual, média histórica e sua anomalia para Barbalha-CE entre 1973 e 2017.

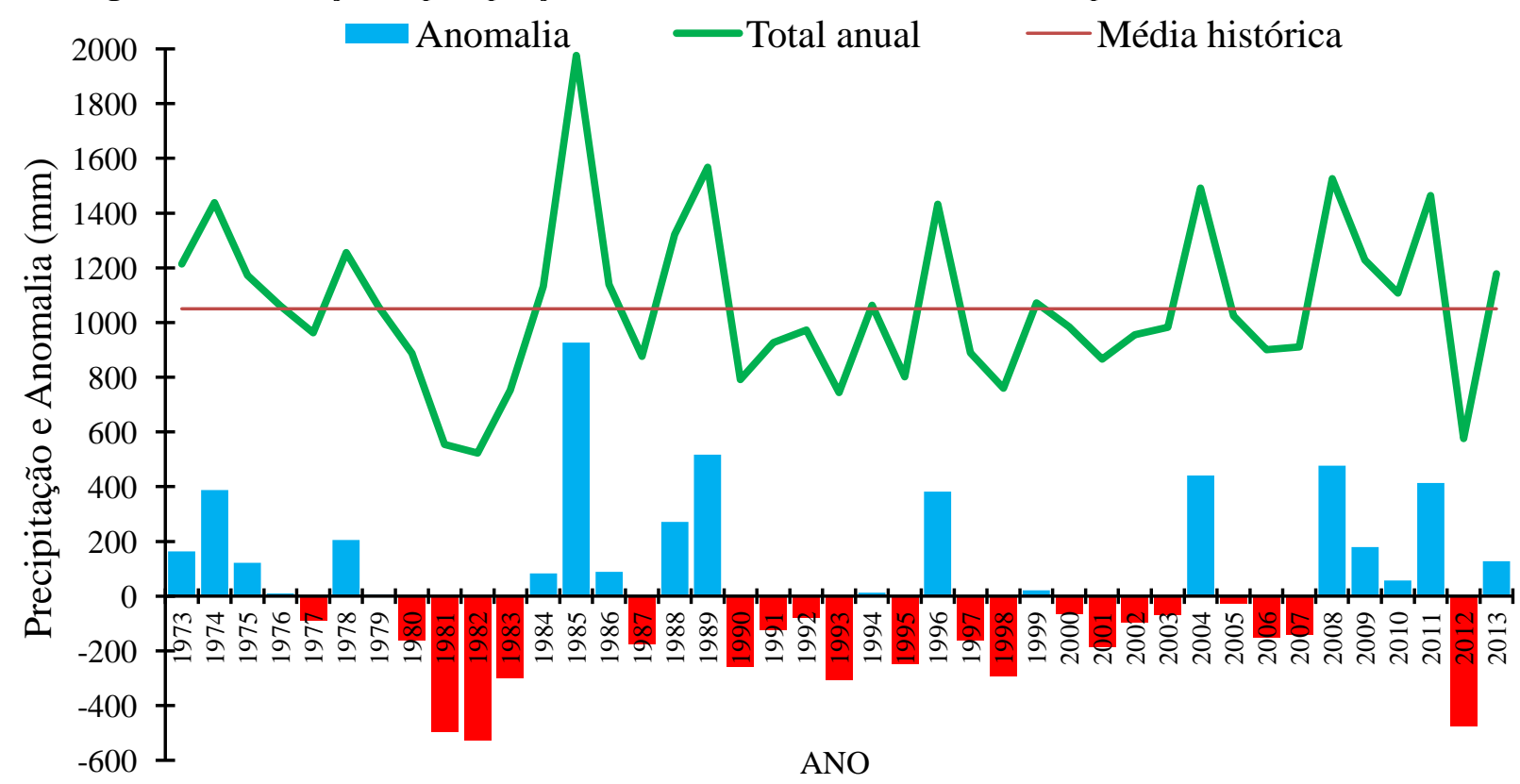

Fonte: Saboya (2021).

A flutuação das anomalias pluviais está em representação na Figura 2. Com chuvas acima da média tem-se 17 anos. Os anos de 1976; 1979; 1994; 1999 foram os menores índices de anomalias positivas. As oscilações positivas de anomalias fluíram entre $75 \mathrm{~mm}$ a $910 \mathrm{~mm}$.

As anomalias negativas oscilaram de 55,5 mm (2005) a $432 \mathrm{~mm}$ em 1982. O estudo de França et al (2020) vem a corroborar com as discussões apresentadas.

No Quadro 2 têm-se as estatísticas descritivas, como: média, erro padrão, mediana, moda, desvio-padrão, coeficiente de variação, curtose, coeficiente assimetria, máximo e mínimo absolutos, nível de confiança a (95\%) e os respectivos anos de ocorrências dos máximos e mínimos valores para os dados de precipitação de 1973-2017, em Barbalha - Ceará, Brasil.

As médias fluem entre 3,6 mm (agosto) a 237,4 mm (março). O quadrimestre de maiores índices pluviais registra-se entre janeiro a abril, e o de menores índices ocorrem entre junho a setembro. A mediana tem oscilação mensal fluindo entre 0,3 $\mathrm{mm}$ (agosto) a 231,3 mm (março). No quadrimestre (janeiro a abril) de maior índice pluvial a mediana oscila de 156,3 mm a 231,3 mm e no quadrimestre junho a setembro suas oscilações são de $0,3 \mathrm{~mm}$ a $8,2 \mathrm{~mm}$. 
Quadro 2. Tem-se os valores mensais da média, erro padrão, mediana, moda, desvio-padrão, coeficiente de variação, curtose, coeficiente assimetria, máximo e mínimo absolutos, nível de confiança a (95\%) e os respectivos anos de ocorrências dos máximos e mínimos valores para os dados de precipitação entre 1973 e 2017, em Barbalha-CE, Brasil.

\begin{tabular}{|c|c|c|c|c|c|c|c|c|c|c|c|c|c|}
\hline $\begin{array}{l}\mathscr{D} \\
\mathbb{N} \\
\stackrel{\infty}{\Sigma}\end{array}$ & 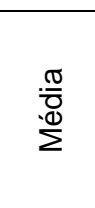 & $\begin{array}{l}\text { 웜 } \\
\frac{\pi}{0} \\
\frac{\pi}{0} \\
0 \\
0 \\
\frac{0}{4}\end{array}$ & 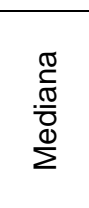 & $\begin{array}{l}\frac{\pi}{8} \\
\frac{0}{2}\end{array}$ & 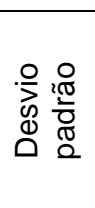 & 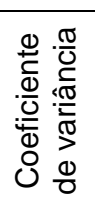 & 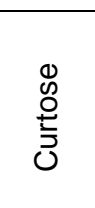 & 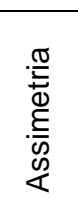 & 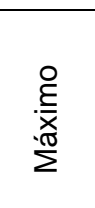 & $\stackrel{\stackrel{ }{E}}{\stackrel{\text { E }}{\Sigma}}$ & 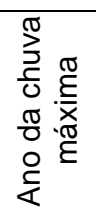 & 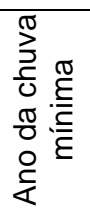 & 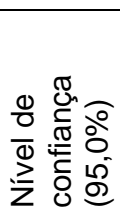 \\
\hline Janeiro & 179,3 & 15,1 & 159,1 & $\# \mathrm{~N} / \mathrm{D}$ & 101,3 & 0,565 & 1,16 & 0,91 & 513,3 & 38,6 & 2004 & 2012 & 30,4 \\
\hline Fevereiro & 200,2 & 16,3 & 156,3 & \#N/D & 109,3 & 0,546 & $-0,51$ & 0,65 & 461,9 & 45,3 & 2004 & 1982 & 32,8 \\
\hline Março & 237,4 & 17,2 & 231,3 & $\# \mathrm{~N} / \mathrm{D}$ & 115,4 & 0,486 & 0,87 & 0,80 & 593,8 & 32,3 & 2008 & 1995 & 34,7 \\
\hline Abril & 181,3 & 17,1 & 165,5 & $\# \mathrm{~N} / \mathrm{D}$ & 114,5 & 0,632 & 1,37 & 1,14 & 533,8 & 20,3 & 1984 & 1980 & 34,4 \\
\hline Maio & 59,3 & 8,7 & 40,1 & $\# \mathrm{~N} / \mathrm{D}$ & 58,3 & 0,982 & 2,82 & 1,64 & 267,9 & 0,0 & 2009 & 1998 & 17,5 \\
\hline Junho & 19,2 & 4,7 & 8,2 & 10,8 & 31,5 & 1,645 & 9,54 & 3,00 & 157,3 & 0,0 & 2013 & 1993 & 9,5 \\
\hline Julho & 17,9 & 5,6 & 6,3 & 0,0 & 37,6 & 2,095 & 28,14 & 4,91 & 238,9 & 0,0 & 2002 & 1980 & 11,3 \\
\hline Agosto & 3,6 & 1,2 & 0,3 & 0,0 & 7,7 & 2,148 & 23,31 & 4,37 & 47,2 & 0,0 & 1973 & 1974 & 2,3 \\
\hline Setembro & 6,7 & 1,7 & 2,0 & 0,0 & 11,6 & 1,744 & 4,72 & 2,28 & 49,0 & 0,0 & 1992 & 1981 & 3,5 \\
\hline Outubro & 22,6 & 5,1 & 13,2 & 0,0 & 34,2 & 1,512 & 18,02 & 3,69 & 204,3 & 0,0 & 2011 & 1987 & 10,3 \\
\hline Novembro & 41,1 & 5,9 & 30,6 & 0,0 & 39,4 & 0,958 & 3,07 & 1,59 & 182,8 & 0,0 & 1996 & 1983 & 11,8 \\
\hline Dezembro & 81,6 & 10,5 & 63,6 & 57,1 & 70,4 & 0,863 & 3,50 & 1,70 & 333,9 & 0,0 & 1985 & 1991 & 21,1 \\
\hline
\end{tabular}

Legenda: (\#N/D) valores não definidos ou encontrados para a moda. Fonte: Saboya (2021).

A média flui entre 3,6 mm (agosto) a 237,4 mm (março), o valor pluvial médio anual da área de estudo distancia-se do valor estatístico da moda (menor concentração dos dados), situando-se a mediana em uma posição intermediaria na distribuição de frequência dos valores pluviométricos, este resultado é confirmado devido ao médio desvio dos valores da média e mediana, a moda nos cincos primeiros meses não determinaram seus valores, nos meses de junho e dezembro registraram-se valores inferiores à mediana e média, indicando grande variação dos dados.

O desvio padrão é um grau de dispersão dos dados relativamente à média, destacam-se irregularidades na dispersão das medidas deste elemento em relação à média, isto é o desvio está mais próxima da média que a mediana. Observa-se que de outubro a janeiro ocorreram os maiores valores de coeficiente de variação, exibindo deste modo a heterogeneidade dos dados destes meses ao longo dos anos. O desvio padrão não se agrega aos dados da média e mediana exceto para o mês de maio. Registra-se altos valores do desvio padrão $(115,4 \mathrm{~mm})$ e do coeficiente de variância (CV\%) no valor de 98,2\%, este fato já era esperado, uma vez que a área em estudo tem clima diferenciado da circunvizinhança. Analisando a variabilidade com base nos valores do $\mathrm{CV}(\%)$, verifica-se que os valores anuais de precipitação apresentaram variação média baixa entre janeiro a março $(\mathrm{CV} \%<0,7 \%)$, média nos meses de junho a outubro (CV\% maior que 1\%) e alta entre novembro e dezembro (CV\% de 0,95 e 0,86) respectivamente indicando que há uma dispersão média entre os valores médios de precipitação na área de estudo. Trabalho como os dos autores (Mello et al., 2016; Carvalho et al, 2009) que estudara a variação espacial da precipitação média do Estado de São Paulo, corroboram com as discussões deste artigo.

O erro padrão supera as expectativas para os meses de dezembro a abril, ultrapassando os limites estabelecidos pela (OMM 1968).

As Medidas de Assimetria e Curtose visam avaliar o nível de normalidade, ou deformação de uma distribuição. (Borges, 2003). As oscilações nos coeficientes de curtose oscilam de -0,51 (fevereiro) a 28,14 (Julho) mostrando sua declividade negativa som achatamento mais longo e com valores extremos posicionados a direita de sua curva. Ao passo que nos valores de assimetria tem-se uma curva com assimetria positiva (assimetria >0) acentuada a direita.

Os valores máximos e mínimos absolutos registrados na área de estudo também podem ser consultados na Tabela seguidamente de seus anos de registros. 


\section{Conclusões}

A relevância da pesquisa e de fornecer informações precisa e segura para os tomadores de decisões governamentais, agricultores e a sociedade em geral. Pois com o entendimento dos dados e do processo climático é possível determinar alternativas visando solucionar os problemas de perda de produtividade agrícola, principalmente, em culturas de sequeiro, que predominam na área de estudo.

As probabilidades das chuvas prováveis possibilitam estratégias de convivência do homem rural e urbano para uma melhor convivência com o período seco e chuvoso e que desta forma venham a projetar cenários futuros visando o gerenciamento e armazenamento dos recursos hídricos.

Em relação a Probabilidade empírica para ocorrências de totais de chuvas anuais o percentual que se destacou foi 50\% e $70 \%$ com possibilidades de chuva superior à média.

\section{Referências}

Ab’saber. A. N. (2003) Caatingas: O domínio dos sertões secos. In: Os domínios de natureza no Brasil: potencialidades paisagísticas. Ateliê Editorial.

Assis. F. N., Arruda. H. V \& Pereira. A. R. (1996). Aplicações de estatística à climatologia: teoria e prática. Editora Universitária. 161.

Ávila. L. F., Mello. C. R \& Viola. M. R. (2009). Mapeamento da precipitação mínima provável para o sul de Minas Gerais. Revista Brasileira de Engenharia Agrícola e Ambiental. 13. 906-915.

Bernardo. S., \& Hill. R. W. (1978) Um modelo para determinação de irrigação suplementar. Revista Ceres. 25(140), $245-262$.

Botelho. V.A. V. A. Ajuste da distribuição gama aos dados de precipitação pluvial do Município de Lavras Estado de Minas Gerais / Valéria Andrade Villela Amarante Botelho - Lavras. UFLA.

Carvalho. J. R. P., Vieira. S. R. \& Grego. C. R. (2009). Comparação de métodos para ajuste de modelos de semivariograma da precipitação pluvial anual. Revista Brasileira de Engenharia Agrícola e Ambiental. 13(4). 443-448.

Dourado Neto. D., Assis. J. P., Timm. L. C., Manfron. P. A., Sparovek. G., Faraway. J., Marsaglia. G., Marsaglia. J., \& Baddeley. A. (2014). Goftest: Classical Goodness-of-Fit Tests for Univariate Distributions. R package version 1.0-2.

Dourado Neto. D., Assis. J. P., Timm. L. C., Manfron. P. A., sparovek. G., \& Martin. T. N. (2005) Ajustes de modelos de distribuição de probabilidade a séries históricas de precipitação pluvial diária em Piracicaba-SP. Revista Brasileira de Agrometeorologia. 13(2), $273-283$.

Franca. M. V., \& Medeiros. R. M. Oscilações pluviais na bacia hidrográfica do Rio Ipojuca (PE) como fonte alimentadora dos sistemas hidrológicos. Revista Mirante (Online). 13, 189 - 202.

Gomes. M. C. R., \& Franca. R. M. (2019) Uso da análise multivariada para subsidiar no monitoramento da qualidade da água subterrânea na bacia sedimentar do Araripe. sul do estado do Ceará. Revista Geociências. 38. 195 - 205

Holanda. R. M., \& Medeiros. R. M. (2019) Classificação climática pelo método de Köppen e Thornthwaite em Bom Jesus do Piauí. Brasil. Revista Pantaneira. 16. $57-68$.

Lucena. R. L., \& Steinke. E. T. (2015) Fatores geográficos e padrões de circulação atmosférica como base à compreensão do clima semiárido quente da região Nordeste. Revista Workshop Internacional sobre Água no Semiárido Brasileiro. 1. 01-07.

Matos. R.M., Silva. P.F., Borges. V.E., Sobrinho. T.G., Dantas Neto. J., \& Saboya. L.M.F. (2018). Potencial agroclimático para a cultura da mangueira no município de Barbalha - CE. Revista Brasileira de Agricultura Irrigada 12. 10.7127/RBAI.V12N100732.

Medeiros. R. M., \& Holanda. R. M. (2019) Classificação climática e balanço hídrico pelo método de Köppen e Thornthwaite do município de Barbalha. Ceará. Brasil. Revista Equador (UFPI). 8(3). 19 - 43. http://www.ojs.ufpi.br/index.php/equador

Mazucheli. J., \& Emanuell. I. P. (2019). Aplicação da Distribuição Nakagami na Análise de Dados de Precipitação. Revista Brasileira de Meteorologia. 34(1). 17.

Mello. Y. R., \& Oliveira. T. M. N. (2016). Análise Estatística e Geoestatística da Precipitação Média para o Município de Joinville (SC). Revista Brasileira de Meteorologia. 31(2). 229-239.

Moura. M. S. B., Sobrinho. J. E., Filho. F. Q. P., Filho. F. X. O. \& Maia. A. J. (2001). Estimativa da evapotranspiração de referência máxima possível para o município de Mossoró - RN. utilizando a distribuição probabilística de Gumbel. Caatinga. 14(1/2). 25 - 30.

Moura. Q. L., Ruivo. M. L. P. \& Rodrigues. H. J. B. (2015). Variação sazonal da população de bactérias e fungos e dos teores de nitrato de amônio do solo nos sítios do LPA e PPBIO na Amazônia Oriental. Revista Brasileira de Meteorologia. 30(3). 265-274.

Sá. I. B., \& Silva. P. C. G. (2010) Semiárido brasileiro: pesquisa. desenvolvimento e inovação. EMBRAPA Semiárido. 
Silva. A. R., Santos. T. S., Queiroz. D. E., Gusmão. M. O., \& Silva. T. G. F. (2017) Variações no índice de anomalia de chuva no semiárido. Journal of Environmental Analysis and Progress. 02(04), 377-384.

Silva. I. N., Oliveira. J. B., Fontes. L. O. \& Arraes. F. D. D. (2013). Distribuição de frequência da chuva para região Centro-Sul do Ceará. Brasil. Revista Ciência Agronômica. 44(3). 481-487.

Silva. R. O. B. (2013). Tendências de mudanças climáticas na precipitação pluviométrica no estado de Pernambuco. Dissertação (Mestrado Engenharia Civil). Universidade Federal de Pernambuco - UFPE. Recife.

Silva. S. A., Lima. J. S. S. \& Bottega. E. L. (2011). Variabilidade espacial da precipitação pluviométrica para o Estado do Espírito Santo utilizando métodos multivariados. Revista Brasileira de Ciências Agrárias. 6(4). 703-790.

Silva. W.L.C., Oliveira. C.A.S. \& Morquelli. W.A. Subsídios para dimensionamento de sistemas de irrigação. In: Congresso Nacional de Irrigação e Drenagem. 8. ABID. Florianópolis. SC. 1988. 1. 535-553.

SUDENE. Superintendência do Desenvolvimento do Nordeste. Semiárido. Disponível em: http://antigo.sudene.gov.br/delimitacao-do-semiarido.

Thornthwaite. C.W. (1948) An approach toward a rational classification of climate. Geographical Review 38. 10. $2307 / 210739$.

Thornthwaite. C.W., \&Mather. J.R. (1955) The water balance. Publication in Climatology 8. Laboratory of Climatology. Centerton. N. J.

Vieira. J. P. G., Souza. M. J. H., Teixeira. J. M., \& Carvalho. F. P. (2015) Estudo da precipitação mensal durante a estação chuvosa em Diamantina. Minas Gerais. Revista Brasileira de Engenharia Agrícola e Ambiental. 14(7). 762-767. 